\title{
Kernos
}

Revue internationale et pluridisciplinaire de religion grecque antique

4 | 1991

Varia

\section{Le symbolisme de l'œuf dans les cosmogonies orphiques}

\section{Spyrodimos Anemoyannis-Sinanidis}

\section{OpenEdition \\ Journals}

Édition électronique

URL : http://journals.openedition.org/kernos/289

DOI : $10.4000 /$ kernos.289

ISSN : 2034-7871

\section{Éditeur}

Centre international d'étude de la religion grecque antique

\section{Édition imprimée}

Date de publication : 1 janvier 1991

Pagination : 83-90

ISSN : 0776-3824

\section{Référence électronique}

Spyrodimos Anemoyannis-Sinanidis, «Le symbolisme de l'œuf dans les cosmogonies orphiques », Kernos [En ligne], 4 | 1991, mis en ligne le 11 mars 2011, consulté le 19 avril 2019. URL : http:// journals.openedition.org/kernos/289; DOI : 10.4000/kernos.289 
Kernos, 4 (1991), p. 83-90.

\section{LE SYMBOLISME DE L'GEUF DANS LES COSMOGONIES ORPHIQUES}

Pour sonder ce symbolisme, il convient tout d'abord de rappeler la structure cosmologique dans laquelle les textes orphiques font surgir l'image de l'œuf. On sait que le recueil des fragments ${ }^{1}$ contient un certain nombre de cosmogonies ou de théogonies dont cinq ${ }^{2}$ sont communément tenues pour authentiquement orphiques ${ }^{3}$. Bien entendu, le problème est de savoir si cette authenticité ne se heurte pas à des difficultés philologiques et herméneutiques dues au caractère tardif des sources. On remarquera néanmoins que, par-delà leurs différences, ces récits obéissent à un même schème : ils distinguent, dans la formation de l'univers, plusieurs stades ou niveaux qui se succèdent généalogiquement et qui sont désignés tantôt par un, tantôt par deux termes ou agents cosmologiques. Seul le premier terme, qui signifie la source primitive, ne provient d'aucun autre ${ }^{4}$ et ne correspond à aucune figure. La comparaison des cinq cosmogonies révèle une certaine similitude entre les différents agents cosmologiques mis en œuvre et l'on peut, à partir de là, tenter de reconstruire une cosmogonie mère d'où auraient dérivé celles que nous identifions comme orphiques. Certes, ces précisions n'éliminent pas les difficultés d'interprétation. La perspective que nous défendrons dans cette étude ne prétend pas effacer ces difficultés, mais entend d'abord clarifier les différents éléments cosmogoniques et, ensuite, proposer une double interprétation, l'une

1 O. KenN, Orphicum fragmenta, Berlin, Weidmann, $1963^{2}$.

2 Cosmogonie selon Hieronymus et Hellanicus chez Damascius, De prim. princ., $123 \mathrm{bis}$ (I, 317,15 - 3197 R.); Fr. 54 Kern; 1 B 13 DK. Cosmogonie Rhapsodique chez Damascius, op. cit., 123 (I, 316, 18 - 317, 14 R.); Fr. 60 Kern; 1 B 12 DK. Cosmogonie selon EudËme chez Damascius, op. cit., 124 (I, 319, 811 R.); Fr. 28 Kern; 1 B 12 DK. Cosmogonie selon APOLLONIOS DE RHODES, Argonautiques, I, 496-498; Fr. 29 Kern; 1 B 16 DK. Cosmogonie selon ALEXANDRE D'APHrodise, In Arist. Metam., N, 1091 b 4 (821, 5s Hayd.); Fr. 107 Kern.

3 Cf. K. Freeman, The Presocratic Philosophers, A Companion to Diels, Fragmente der Vorsokratiker, Oxford, Blackwell, 1966' ${ }^{2}$, p. 5-6.

4 Fr. 59 Kern. Simplicius, In Arist. De Caelo, III, 1, 298 b 24 (560, 19s Heib.). 
interne au texte, l'autre comme anticipation à la pensée platonicienne. Nous avons conjecturé naguère la structure primitive suivante ${ }^{5}$ :

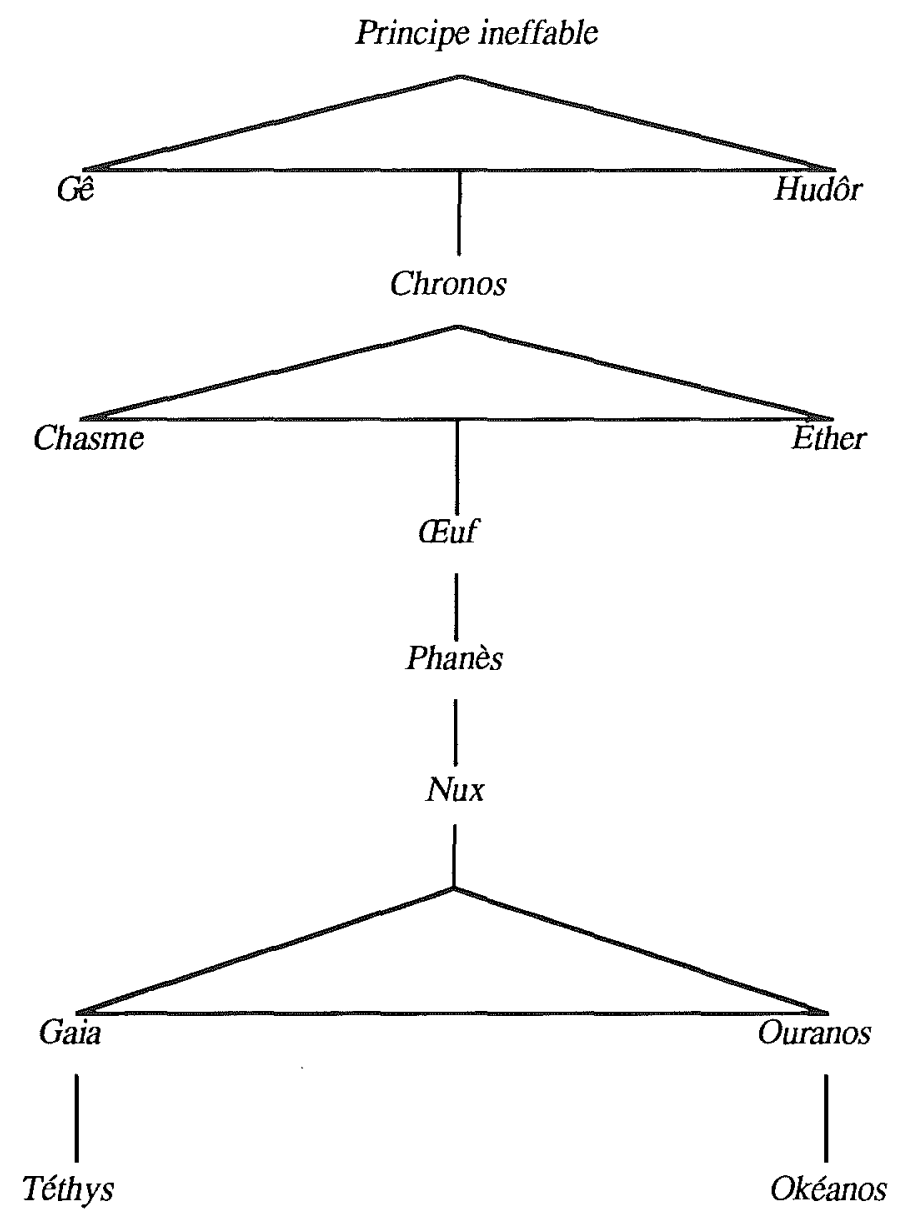

Le premier moment généalogique que je qualifie de "premier niveau" est le "Principe ineffable» ${ }^{6}$; il est cosmologiquement indéfini,

5 Cf. S. Anemoyannis-Sinanidis, Notions du temps et de l'espace dans les fragments orphiques (en grec), Dissertation doctorale de l'Univ. d'Athènes, 1978, p. 19-22.

6 Fr. 54 Kern. - Cette terminologie ne reflète-t-elle pas une contamination du néoplatonisme tardif ? C'est probable. Mais cela ne modifie pas le fond du problème, qui suppose comme origine un indéfini non manifesté. 
non manifesté et contient, ontologiquement encore indéterminés, les deux agents cosmogoniques primitifs qui sont exprimés par les figures de Gè (Terre) et de Hudôr (Eau) $)^{7}$. En sortant du "Principe ineffable», ceux-ci manifestent leurs propriétés et forment le deuxième niveau. Leurs transformations successives en même temps que leurs collaborations renouvelées détermineront plusieurs niveaux ultérieurs : Gè se transforme premièrement en Chasme ${ }^{8}$, puis en Gaia, puis en Téthys, tandis que Hudôr devient parallèlement Éther, Ouranos et enfin Okéanos. A chaque fois se reconstituent de nouveaux couples.

Mais entretemps intervient tout d'abord le fameux Chronos orphique qui forme le troisième niveau. $\mathrm{Si}$, dans son rôle d'intermédiaire, il n'est mentionné qu'une seule fois - avant la première transformation de Gè-Hudôr en Chasme-Éther -, c'est sans doute en raison d'un principe d'économie qui a présidé à l'élaboration de cette cosmogonie primitive. On peut penser que sa présence est supposée tout au long du processus de transformations que subit le couple originel.

Dans la structure primitive que nous conjecturons, l'apparition de $l^{\prime}$ Euf cosmogonique ${ }^{9}$ marque une autre phase intermédiaire qui intervient précisément après l'apparition du couple formé par les deux

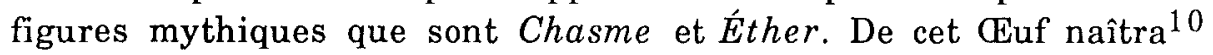

\section{Ibid.}

8 Fr. 66 Kern. Simpl., In Arist. Phys., IV, 1, 208 b 29 (I, 528, 12s DK). La cosmogonie orphique selon HIERONYMUS et HELLANICUS se réfêre aux agents cosmogoniques Ether et Chaos en tant que constituants du quatrième niveau cosmogonique. Étant donné que, selon le fr. 66 Kern (= Proclos, In Plat. Remp., II, 138, 8s Kr.), Chronos a donné naissance à l'Ether et au Chasme et, pour éviter l'insertion du terme Chaos au quatrième niveau - puisque ce terme se réfêre au premier niveau cosmogonique -, le terme Chaos du quatrième niveau a été remplacé légitimement par celui de Chasme. (cf. M.L. WEST, The Orphic Poems, Oxford, Clarendon, 1983, p. 70). Selon l'explication que SimpLICIUS, op. cit., IV, 1, 208 b 29 (I, 528 12s DK) donne (fr. 66 Kern), il paraît qu'à l'époque d'HésIODE, le Chaos était conçu surtout en tant qu'espace précosmique. Cependant, poursuit Simplicius, ce terme ne laisse pas entendre un espace, mais se réfêre à la cause (aitian) formatrice des dieux, de nature infinie (apeiroeidé) et multiple (peplèthusménèn), qu'OrPHÉE désigne par le nom de Chasme énorme.

9 Cf. W.K.C. GuThRIE, A History of Greek Philosophy, I, Cambridge, Univ. Press, 1962, p. 69, et I. LiNFORTH, The Arts of Orpheus, Berkeley, 1941, p. 277.

10 Fr. 85 Kern = DAMASC., op.cit., 111 (I, 286, 15s R.). 
Phanès ${ }^{11}$ ou Éros ${ }^{12}$, qui forme un sixième moment généalogique et donc un sixième niveau. Et c'est ici peut-être que se manifeste le message le plus important de la cosmogonie orphique : c'est une sorte de dieu personnel qui naît ${ }^{13}$ de l'CEuf.

Mais examinons d'abord de plus près la nature des deux agents cosmogoniques, Chasme-Éther, qui donnent naissance à celui-ci : Hiéronymus et Hellanicus, cités par Damascius, apportent au sujet du couple primitif Gè-Hudôr d'intéressantes précisions; ils désignent le premier élément comme un agent de nature "divisée» 14 tandis que l'autre serait de nature "contenante» et "collante» 15 . Comme chez les philosophes, les deux agents cosmogoniques sont ainsi complémentaires, la nature de l'eau se caractérisant par une continuité prononcée. Ces propriétés des deux agents cosmologiques primordiaux se retrouvent naturellement dans leurs concrétisations successives. Chasme ${ }^{16}$, dont le nom indique à suffisance la nature "divisée», est constitué d'un nombre infini de particules infinitésimales; du point de vue de sa nature et de son rôle cosmogonique, il est à rapprocher de la notion platonicienne d'«infini» (apeiron) ${ }^{17}$. La nature "contenante» et surtout "collante» d'Éther implique en revanche une action formatrice qui évoque celle du peras platonicien ${ }^{18}$. On peut songer parallèlement à rapprocher le Chasme orphique de l'Autre platonicien : dans cette nature «divisée», chaque particule implique une altérité par rapport à toutes les autres. $E$ ther, qui est continu et qui est "même" en toutes directions de l'étendue, évoque de son côté la catégorie platonicienne du to auto. Dès lors, l'Euf orphique, issu du couple Chasme-Éther apparaît comme l'analogue de l'âme que le Timée montre composée de la substance du même et de l'autre ${ }^{19}$. Plusieurs témoignages soulignent effectivement

11 Fr. 72 Kern. Cf. W. BuRkeRT, Lore and Science in Ancient Pythagorism, transl. by E.L.Jr. Minar, Cambridge, Massachusetts, Harvard Univ. Pr., 1972, p. 39; W.K.C. GUTHRIE, op.cit, t. 1, p. 69.

12 Fr. 74 Kern. Cf. M.P. NILsson, Geschichte der griechischen Religion, I, München, Beck, 2e éd., p. 685.

13 Fr. 59 Kern = SrMPL., In Arist. De Cælo, III, 1, 298 b 24, p. 560, 19s Heib.

14 Fr. 54 Kern.

15 Ibid.

16 Fr. 66 Kern = ProCL., op. cit., II, 138, $8 \mathrm{~s} \mathrm{Kr.}$

17 Fr. 66 \& 79 Kern. Cf. M.L. WeST, op. cit., p. 199.

18 Fr. 66 Kern.

19 Timée, 34b10-35b3. Cf. fr. 54 Kern = DAMASc., op. cit., 123bis (I 318, 14-18 R.); fr. 66 Kern = Proclos, In Plat. Tim., 30c-d (I 428, 4-9 Diehl). Cf. supra, n. 8, pour le terme de Chaos à ce quatrième niveau. 
le caractère animé de l'œuf cosmogonique ${ }^{20}$, ce qui ne peut surprendre puisque c'est de lui que sort ${ }^{21}$ le dieu tout resplendissant nommé Phanès ou Éros. Bref, tout porte à croire que nous assistons ici à une transformation de la démarche généalogique archaïque en structure philosophique où les figures mythiques sont en même temps comprises comme des principes constitutifs des processus et des choses, et hiérarchiques. C'est ce qui ressort plus clairement encore de ce qui suit.

En effet, une autre donnée, plus importante encore, est à souligner pour la compréhension de l'orphisme. La nature matérielle du couple d'agents cosmogoniques Gè-Hudôr ${ }^{22}$, comme de son dérivé ChasmeÉther, implique que l'âme elle-même, dans son principe, est aussi matérielle; son immortalité dérive dès lors du caractère indestructible des éléments qui la composent ${ }^{23}$. Selon la cosmogonie orphique, l'âme est donc une chose de la nature et le divin personnel provient de la nature matérielle; le monde matériel est lui-même une manifestation de la nature divine, laquelle est issue de la réalité divine et matérielle qui, coïncidant avec le premier niveau, est indéfinie et nonmanifestée ${ }^{24}$. On ne doit pas perdre ici de vue les restrictions sévères qu'imposait la discipline religieuse du secret à l'évocation de la nature divine des agents cosmogoniques, restriction qui permet aussi d'expliquer la variété des termes utilisés par les premiers philosophes pour désigner les agents cosmiques qu'ils disent prépondérants 25 .

Dans la structure de la cosmogonie orphique primitive, telle que nous l'avons conjecturée, il apparaît ainsi que l'élément matériel et impersonnel représenté par le couple Gè-Hudôr et l'élément personnel que signifie le dieu Phanès sont très étroitement associés. Il convient

20 Fr. 56 Kern = APION apud Clem. Rom., Homil., VI, 5-12 (Migne, P.L., II, col. 200).

21 Fr. 54 Kern = DAMASC., op. cit., 123bis ( I 317, 15s R.); fr. 56 Kern = APION apud CLEM. ROM., op. cit, VI, 5-12s; fr. 60 Kern = DAMASC., op. cit., 123 (I 316, 18s R.).

22 Fr. 54 Kern = DAMASC., op. cit., 123bis (I 317, 16-17 R.); fr. 55 Kern = APION apud Clem. RoM., op. cit., VI, 3, 4 (Migne, P.L., II, col. 198); fr. 353 Kern = OLYMP., In Plat. I Alcib., p. 19 Creuz; cf. M.L. WEST, op. cit., p. 200.

23 Fr. 95 Kern = Proclos, op. cit., $21 \mathrm{~d}$ (I 94, 13 Diehl).

24 Fr. 66 Kern = Proclos, op. cit., 30a (I 385, 29s Diehl).

25 Cf. O. CASEL, De philosophorum Graecorum silentio mystico, Berlin, Töpelmann, 1967, p. 88, à propos de PLUTARque apud EusèBe, Pr. Ev., III, 1, éd. Bernardakis VII, p. 43; cf. aussi M.L. WEST, op. cit., p. 80, et S. ANEMOYANNISSinanidis, Interprétations de l'ontologie du Sophiste de Platon [en grec], Athènes, 1981, p. 166. 
d'insister cependant sur le fait que l'élément matériel précède et suit ${ }^{26}$ le stade où apparait la figure personnelle et énigmatique de Phanès, et qu'il reste donc présent aussi à ce niveau.

Ces préliminaires ont mis en évidence la position qu'occupe l'Euf dans la cosmogonie orphique et laissent déjà pressentir certains aspects du symbolisme complexe qui s'y attache.

Né de la rencontre entre les particules de Chasme et la continuité de l'Éther, l'Guf représente, en un modèle aussi réduit et aussi économique que possible, l'unité ${ }^{27}$ que forme la réalité matérielle ${ }^{28}$. Il est une sorte de répétition cosmogonique du "Principe ineffable» 29 : en lui sont unis Chasme et $\hat{E}$ ther, tout comme Gè et Hudôr l'étaient dans le Premier principe. Tel apparaît donc le précurseur de Phanès perçu sous son aspect impersonnel ${ }^{30}$.

Mais le fait que cette unité se transforme et devient le dieu personnel qu'est Phanès ou Éros implique que l'Euf orphique symbolise également l'âme, laquelle est bien conçue par ces traditions comme une réalité matérielle, mais animée ${ }^{31}$. Les fragments ne conservent cepen-

26 La "Nuit» orphique est elle-même un espace, rempli d'une continuité matérielle en tant que substance; fr. 131 Kern = DAMASC., op. cit., 67 (I 146, 12-17 R.). Cf. S. ANEmoyannis-Sinanidis, Notions de l'espace..., p. 78-79. Si les Orphiques avaient voulu signifier la nature «spirituelle» des agents du deuxième niveau, ils auraient pu trouver aisément dans la langue grecque les termes adéquats qui auraient suggéré cette qualité (cf. ibid., p. 38).

27 Fr. 60 Kern = DAMASC., op. cit., 123 (I 316, 18 R.).

28 Cf. ARIST., Metaph., N, 3, 1091 a12 : le premier hen, selon les Pythagoriciens, proviendrait de la collaboration reproductive des deux agents cosmogoniques.

29 Fr. 79 Kern = PROClOS, op. cit., 30c-d (I 427, 20s Diehl); fr. 55 Kern = APION apud Clém. Rom., op. cit., VI, 3, 4 (Migne, P.L., II, col. 199); fr. 81 Kern = Proclos, op. cit., 30c-d (I 429, 26s Diehl).

30 Fr. 170 Kern = PRoclos, op. cit., 29a-b (I 336, 6-16 Diehl).

31 Fr. 60 Kern = DAMASC., op. cit., 123 (I 317, 2-4 R.); fr. 56 Kern = APIoN apud CLÉm. RoM., op. cit., VI, 5-12 (P. DE LAGARDE, Clementina, 77, 7s). Que l'œuf soit symbole de l'âme correspond sans doute à l'état le plus ancien de la pensée orphique. Les textes (Kern, p. 95-97) qui font état d'un transport de l'âme par les vents proviennent de spéculations plus tardives, car l'idée s'accommode mal avec la conception qui se dégage de la cosmogonie primitive considérée dans sa structure. Si l'on admet dès lors, avec PhILopon (186, 24 Hayd = fr. 27 Kern), qu'ONOMACRITE avait professé cette idée, ce serait là un indice que les 
dant nulle trace de la manière dont les Orphiques concevaient la transformation en âmes des particules infinitésimales de Chasme, enveloppées d'Éther, chacune étant un Euf. Sans doute était-ce un secret théologique. Mais cela n'empêche pas de constater que la structure généalogique du récit recouvre un mythe d'origine axé sur l'âme et sur tout ce que celle-ci suppose pour la secte.

Qu'on nous permette ici de tenter un rapprochement entre l'Euf orphique et le symbolisme de l'omphalos. On sait qu'un objet ainsi nommé se trouvait représenté sur des vases sacrés évoquant certaines scènes des mystères éleusiniens ${ }^{32}$, antiques cérémonies visant à élever l'âme vers le divin. Que cet objet ait pu être transféré du sanctuaire apollinien de Delphes, où l'on peut encore en voir un exemplaire taillé dans une pierre ovale ${ }^{33}$, au culte des «deux déesses» d'Éleusis indique à suffisance que la signification symbolique de "centre de la terre» qu'on lui reconnaissait à Delphes n'était pas exclusive. Par ailleurs, la forme semi-sphérique ou semi-ovoïde qu'ont généralement les représentations de l'omphalos ne font guère penser à un nombril humain. Le symbolisme religieux archaïque se montre d'ordinaire beaucoup plus rigoureux dans les équivalences qu'il institue. On est ainsi amené à penser que l'omphalos a pu être une figuration de l'Euf orphique, et qu'il voilait ainsi une référence à l'âme, que concernaient directement les mystères d'Éleusis et les rites sacrés de Delphes.

Quoi qu'il en soit de cette hypothèse, il apparaît que le passage du cinquième niveau $\mathscr{E} u f$ au sixième Phanès signifie, sur le plan personnel et initiatique, le mouvement de l'âme vers le divin ${ }^{34}$. Ici encore cependant, les textes orphiques conservés se gardent de dévoiler le processus de cette déification.

De cet essai de reconstitution de la cosmogonie primitive des Orphiques et d'analyse que nous en avons proposé, on retiendra surtout le fait que l'Guf occupe, dans cette structure, une position qui le donne

cosmogonies orphiques sont d'époque qui lui est bien antérieure. Cf. M.L. WEST, op. cit., p. 69-70 et 125, et G.S. KIRK-J.E. RAVEN-M. SCHOFIELD, The Presocratic Philosophers, Cambridge, Univ. Pr., 19832, p. 26-29.

32 Cf. J. Harrison, Prolegomena to the Study of Greek Religion, London, Merlin Press, 1980, p. 556 et 558; M.P. NILSSON, op. cit., p. 126, n. 4.

33 Cf. M.P. NILsson, op. cit., p. 204 et 558; J. HARRISON, op. cit., p. 390.

34 Fr. 30 Kern = CHRYSIPPE apud PHILODÈme, De pietate, 80, 14s Gomp.; fr. 32c Kern, p. 107 : cf. D. CoMPARETTI, Laminette Orfiche, Firenze, 1910, p. 17; fr. $32 \mathrm{f}$ Kern : D. CompareTti, op. cit., p. 6; fr. 57 Kern = ATHÉnag., Pro Christianis, 18, p. 20, 12 Schw.; Fragmenta Aristotelica, 48, 1483b17; saint JEAN, X, 34-37; G.S. KIRK-J.E. RAVEN-M. SCHOFIELD, op. cit., p. 33. 
comme postérieur à l'apparition des deux agents de nature matérielle

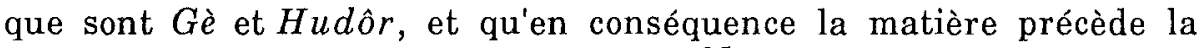
manifestation de l'intellect ou de l'esprit ${ }^{35}$. Phanès lui-même est bien de nature matérielle, qu'il s'agisse de son côté impersonnel, en tant qu'il est une transformation de Zeus-Éther ${ }^{36}$, ou de son côté personnel, en tant qu'il est une âme divine dérivée de l'Guf cosmogonique et perceptible seulement par cette enveloppe resplendissante que sont la néphélè, la «nuée», ou le chiton $^{37}$, la «tunique», tous deux éthérés. Dans les manifestations successives du divin impersonnel ${ }^{38}$ contenu dans le «Principe ineffable», l'Guf orphique représente le chaînon décisif d'une transformation en une multitude d'unités divines personnelles que désigne le terme collectif Phanès.

34/36, rue Karnéadou

Spyrodimos ANEMOYANNIS-SINANIDIS

GR - 10676 ATHÈNES.

35 Fr. 107 Kern = Alex. APHrod., In Arist. Met., N, 1091b4 (821, 5s Hayd.). Cependant, cf. ARIST., op. cit., N4, 1091b4; L6, $1071 \mathrm{b27.}$

36 Fr. 168 Kern = Proclos, op. cit, 32c-d (II 54, 28-30 Diehl), 41a (II 208, 29-209, 6 Diehl). Cf. E. Zeller-R. Wondolfo, La filosofia dei Greci, I, t. 1, Firenze, La Nuova Italia, 1967, p. 160.

37 Fr. 60 Kern = DAMASC., op. cit., 123 (I 316, 18s R.).

38 Fr. 297 Kern = IOAN. DiaCON. GALEN., Hésiode Théogon., 943 (II 604, 3s Gaisf.); cf. Proclos, In Plat. Alcib., 109e, p. 509, 9-18 Cous. Fr. 168 Kern = PORPHYRE apud EusèBe, Pr. Evv., III, 9, p. 100a-105d (I 121, 12s Dind.). Esch., fr. 70 N2. 